\title{
Percepção de discentes, docentes e técnicos em enfermagem a respeito dos eventos adversos
}

\author{
Perception of nursing students, teachers and technicians regarding adverse events \\ Percepción de estudiantes de enfermería, docentes y técnicos sobre eventos adversos
}

\section{Neiva Claudete Brondani Machado , Edinara Moraes Morais ${ }^{\mathrm{II}}$, Rosane Teresinha Fontana ${ }^{\mathrm{III}}$ Andressa Peripolli Rodrigues ${ }^{\mathrm{IV}}$, Miriam de Barros ${ }^{\mathrm{v}}$, Elizabet Marta Krebs ${ }^{\mathrm{vI}}$}

Resumo: Objetivo: identificar a percepção de discentes, docentes e técnicos em enfermagem a respeito dos eventos adversos. Método: pesquisa descritiva, exploratória, qualitativa, realizada em instituição de ensino básico, técnico e tecnológico localizada no noroeste do Rio Grande do Sul. Os participantes foram discentes, docentes e técnicos em enfermagem. Os dados foram coletados com entrevista semiestruturada e analisados pela análise de conteúdo. Resultados: participaram do estudo 25 técnicos em enfermagem, 24 discentes e quatro docentes. A sobrecarga de trabalho e a demanda de cuidados contribuem para que a ocorrência de algum evento adverso se torne iminente. Além disso, a falta de atenção e as falhas no processo de formação são citadas como possíveis condicionantes à essa problemática. Considerações finais: perceber diferenças cruciais entre discentes e profissionais formados no que se refere aos eventos adversos permite uma comunicação essencial para (re) descobrir formas de ensinar enfermagem de maneira segura e de qualidade.

Descritores: Capacitação profissional; Segurança do paciente; Cuidados de enfermagem; Dano ao paciente; Enfermagem

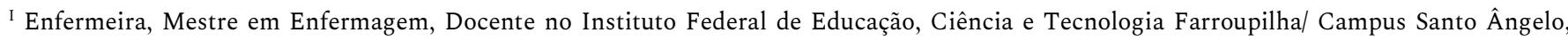
Santo Ângelo, Rio Grande do Sul, Brasil. Email: neiva.machado@iffarroupilha.edu.br ORCID: https://orcid.org/0000-0002-5260-9665

II Enfermeira, Mestre em Atenção Integral à Saúde, Enfermeira Assistencial no Instituto Federal de Educação, Ciência e Tecnologia Farroupilha/ Campus Santo Ângelo, Santo Ângelo, Rio Grande do Sul, Brasil. Email: edinara.morais@iffarroupilha.edu.br ORCID: https://orcid.org/0000-0002-4092-5046

III Enfermeira, Doutora em Enfermagem, Docente na Universidade Regional Integrada do Alto Uruguai e das Missões (URI)/ Campus Santo Ângelo, Santo Ângelo, Rio Grande do Sul, Brasil. Email: rfontana@san.uri.br ORCID: https://orcid.org/0000-0002-0391-9341

IV Enfermeira, Doutora em Enfermagem, Docente no Instituto Federal de Educação, Ciência e Tecnologia Farroupilha/ Campus Santo Ângelo, Santo Ângelo, Rio Grande do Sul, Brasil. Email: andressa.rodrigues@iffarroupilha.edu.br ORCID: https://orcid.org/0000-0002-7599-4674

V Técnica em Enfermagem, Unimed Missões, Santo Ângelo, Rio Grande do Sul, Brasil. Email: miriam.debarros@hotmail.com ORCID: https://orcid.org/0000-0002-4917-3952

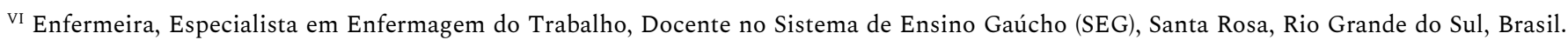
Email: betikrebs@hotmail.com ORCID: https://orcid.org/0000-0003-1527-3960
} 


\begin{abstract}
Aim: To identify the perception of nursing students, teachers and technicians regarding adverse events. Method: descriptive, exploratory, qualitative research, carried out in a primary, technical and technological institution located in the northwest of Rio Grande do Sul. The study participants were students, teachers and nursing technicians. Data were collected with semi-structured interviews and analyzed by content analysis. Results: 25 nursing technicians, 24 students and four teachers participated in the study. Work overload and demand for care contribute to the occurrence of any adverse event imminent. In addition, lack of attention and failures in the training process are cited as possible constraints to this problem. Final considerations: Understanding crucial differences between students and graduates regarding adverse events allows for essential communication to (re) discover ways to teach nursing safely and in a quality way.
\end{abstract}

Descriptors: Professional training; Patient safety; Nursing care; Patient harm; Nursing

Resumen: Objetivo: identificar la percepción de los estudiantes de enfermería, docentes y técnicos sobre los eventos adversos. Método: investigación descriptiva, exploratoria, cualitativa, realizada en una institución primaria, técnica y tecnológica ubicada en el noroeste de Rio Grande do Sul. Los participantes fueron estudiantes, maestros y técnicos de enfermería. Los datos fueron recolectados con entrevistas semiestructuradas y analizados por análisis de contenido. Resultados: 25 técnicos de enfermería, 24 estudiantes y cuatro maestros participaron en el estudio. La sobrecarga de trabajo y la demanda de atención contribuyen a la aparición de cualquier evento adverso inminente. Además, la falta de atención y las fallas en el proceso de capacitación se mencionan como posibles limitaciones para este problema. Consideraciones finales: comprender las diferencias cruciales entre los estudiantes y los graduados con respecto a los eventos adversos permite una comunicación esencial para (re) descubrir formas de enseñar enfermería de manera segura y de calidad.

Descriptores: Capacitación professional; Seguridad del paciente; Atención de enfermeira; Daño del paciente; Enfermería

\title{
Introdução
}

O ato pedagógico é extremamente relevante para o processamento da informação trabalhada em sala de aula, uma vez que as explicações e a atuação docente não somente informam como também oferecem dados para reflexões, os quais, colhidos nas interações vivenciadas, transformam-se em momentos de aprendizagem. Assim, gesto e fala fornecem mensagens significativas a serem decodificadas. ${ }^{1}$

Nesse sentido, promover a aprendizagem exige dos professores o conhecimento fundamental e a capacidade de estimular o seu próprio potencial e o potencial dos educandos. ${ }^{2}$ Com o passar do tempo, as exigências sociais se modificaram e os anseios profissionais também, fazendo com que o ensino se volte para o desenvolvimento de competências, para aceitação das diversidades e desenvolvimento de senso crítico, envolvendo um saber reflexivo. 
Além disso, a renovação teórica na formação docente com vistas a um momento de transformação diante das lacunas curriculares requer reflexão e ação. É necessário um currículo que atenda aos interesses profissionais de aprendizado, que trabalhe as competências de maneira dialógica em uma perspectiva de aprendizagem, que não desconsidere a subjetividade do educando e indique ação para a aprendizagem baseada na prática profissional e nas evidências científicas. ${ }^{3}$

Diante desse contexto, a enfermagem como ciência busca, por meio do cuidado, proteger a vida em sua plenitude, depara-se com uma realidade que exige qualificação no processo de formação dos profissionais da categoria. A discussão sobre os eventos adversos na enfermagem e sua multicausalidade ocupa o centro da atenção de uma profissão que, historicamente, tem um legado importante no cuidado aos indivíduos. ${ }^{4}$

Evento adverso é o incidente que resulta em dano ao paciente, e este incidente se refere ao evento ou circunstância que poderia ter resultado, ou resultou, em dano desnecessário ao paciente. ${ }^{4}$ Desse modo, é imprescindível refletir sobre o crescimento excessivo deste tipo de evento na atuação dos profissionais de enfermagem e suas implicações para a sociedade e para a credibilidade da profissão.

Estudo de revisão que incluiu estudos nacionais e internacionais identificou que os principais eventos na assistência de enfermagem estão relacionados aos erros na administração de medicação, não realização de curativos e as quedas. Também destacou que são necessários instrumentos de notificação de eventos adversos nas instituições de saúde; no entanto, o medo dos profissionais a respeito de uma possível punição pode contribuir para a subnotificação. ${ }^{5}$

Neste contexto, emerge a necessidade de discussão a respeito dos fatores determinantes de tais eventos e o debate em sala de aula. Assim, é possível suscitar, nos docentes e discentes, reflexão para uma atenção profunda sobre estas questões que colocam em risco a integridade e a 
Percepção de discentes, docentes e técnicos em enfermagem a respeito dos eventos adversos |4

vida dos usuários dos serviços de saúde, visando impedir e/ou minimizar a incidência de eventos adversos.

Os docentes de enfermagem enfrentam o desafio de contribuir para a formação de um profissional que atue com competência no mercado de trabalho, com postura crítica e que seja capaz de acessar e utilizar o conhecimento como um agente de mudanças, superando os obstáculos. Esse perfil da enfermagem demanda, além de competências técnicas específicas, competências gerenciais para sua atuação nas organizações de saúde, sendo elas: atenção à saúde, tomada de decisão, liderança, trabalho em equipe, comunicação, educação permanente e gestão. ${ }^{6}$

É válido destacar que alguns estudos apontam que os eventos adversos estão relacionados à administração de medicamentos. ${ }^{7-9}$ No entanto, conforme relatório de eventos notificados à Agência Nacional de Vigilância Sanitária (ANVISA) entre os anos de 2014 a 2019, 21.759 notificações são referentes a perdas de cateteres ou sondas, seguido de flebites, notificações diversas, notificações envolvendo cateteres periféricos e em $5^{\circ}$ lugar as notificações envolvendo medicamentos. ${ }^{10}$ Assim, evidencia-se a necessidade de discussão profunda a respeito da segurança dos usuários dos serviços de saúde e das condições de formação e de trabalho dos profissionais envolvidos no cuidado. ${ }^{7-9}$

Diante disso, questiona-se qual a percepção de discentes, docentes e técnicos em enfermagem a respeito dos eventos adversos? Tem-se como objetivo do presente estudo identificar a percepção de discentes, docentes e técnicos em enfermagem a respeito dos eventos adversos.

\section{Método}

Estudo exploratório e descritivo, com abordagem qualitativa, realizado de junho a agosto de 2016, em uma instituição de ensino básico, técnico e tecnológico localizada na região noroeste do Rio Grande do Sul (RS). A população incluída foi selecionada por meio de 
amostragem não probabilística por conveniência, em que foram contemplados docentes e discentes do curso técnico em enfermagem da referida instituição e técnicos em enfermagem que atuavam nos turnos da manhã e tarde em um hospital filantrópico na referida região, que atuavam em cinco unidades hospitalares do referido serviço: cirúrgica, clínica médica, bloco cirúrgico, ambulatório e recuperação anestésica.

Os critérios de inclusão para participação no estudo foram: discentes do curso técnico em enfermagem, com regular frequência nas aulas; técnicos em enfermagem em exercício ativo nas unidades hospitalares; e docentes supervisores de atividades práticas/estágio curricular. Foram excluídos do estudo: discentes ingressantes no curso ( $1^{\circ}$ semestre).

A amostra foi composta de 24 discentes, 25 técnicos em enfermagem e quatro docentes. Destaca-se que não houve perdas no estudo, uma vez que se trabalhou com a totalidade dos participantes. A coleta de dados ocorreu por meio de entrevista semiestruturada, realizadas individualmente e gravadas de acordo com a autorização dos participantes, tendo duração aproximada de 30 minutos.

O instrumento utilizado na entrevista foi constituído de duas partes, uma contendo a caracterização dos entrevistados com idade, sexo, estado civil, número de filhos, motivos pela escolha da profissão e local de atuação; e a outra em que se questionava a ocorrência de eventos adversos e quais fatores contribui para essas ocorrências.

A coleta dos dados ocorreu em sala reservada para esse fim, na instituição de ensino e no ambiente hospitalar. Com os discentes os dados foram coletados nos intervalos das aulas, para que estes não fossem prejudicados; da mesma forma, com os docentes, ocorreu durante o período em que não estivesse em atuação direta em sala de aula. Com os profissionais ocorreu durante a jornada de trabalho, no momento em que este sinalizava ser propício para a entrevista e que não intervisse no cuidado e na sua rotina de trabalho. 
Percepção de discentes, docentes e técnicos em enfermagem a respeito dos eventos adversos $\mid 6$

Os dados foram analisados por meio da análise de conteúdo, que é uma técnica de levantamento de dados que utiliza textos, falas, informações já coletadas, de forma extensiva. Sua proposta é analisar, em maior profundidade, a pertinência das respostas, a lógica, a coerência, a fidedignidade dos dados informados, eventuais distorções e omissões voluntárias. ${ }^{11}$ Atribuiu-se aos participantes do estudo da pesquisa nomes fictícios de flores para garantir o seu anonimato.

A pesquisa teve início após aprovação do Comitê de Ética em Pesquisa em março de 2016, sob parecer número 1.470 .720 e desenvolvida após a autorização dos gestores dos campos em que a coleta dos dados foi realizada, com assinatura do termo de ciência e de coparticipação. Foram respeitados os aspectos éticos para a pesquisa envolvendo seres humanos, estabelecidos pela resolução n⿳0 466 de 2012, ${ }^{12}$ utilizando-se o Termo de Consentimento Livre e Esclarecido aos que aceitaram participar do estudo, sendo entregue em duas vias, ficando uma com o entrevistado e a outra com o pesquisador.

\section{Resultados e discussão}

Dos 24 discentes participantes, 71\% (n=17) possuem idade entre 21 e 50 anos, 79\% (n=19) é do sexo feminino, $50 \%(n=12)$ com união estável e $58 \%(n=14)$ possuem um ou mais filhos. Com relação aos técnicos em enfermagem, 88\% (n=21) tinha idade entre 21 e 50 anos, 92\% (n=22) do sexo feminino, $63 \%(n=15)$ união estável e $83 \%(n=20)$ possuem um ou mais filhos. Dos docentes, todos $(\mathrm{n}=4)$ apresentaram idade entre 21 e 50 anos, do sexo feminino, 75\% (n=3) união estável e $(\mathrm{n}=4)$ não possuem filhos.

Observa-se, a partir destes dados, que a enfermagem predominantemente é formada por profissionais do sexo feminino. Historicamente, essa realidade é comum nos serviços de saúde, percebendo-se que o cuidado é um elemento que faz parte da vida das mulheres e, por isso, o número elevado delas na área. ${ }^{13}$ Além da predominância feminina, os dados apontam que a 
maioria dos discentes e profissionais possuem um ou mais filhos, o que já pode contribuir para uma sobrecarga maior no trabalho, tendo como ponto de partida o lar.

Com relação à escolha da profissão, os discentes quando questionados a respeito do motivo que os levaram à enfermagem relataram:

Qualificação e facilidade de inserção no mercado de trabalho. (ROSA)

Pensava que poderia trabalhar somente um turno do dia. (TULIPA)

Trabalhar em mais de um lugar e ter uma renda maior. (CRAVO)

A escolha de uma profissão repercute na vida do estudante e, normalmente, está influenciada por familiares, pelas expectativas favoráveis de inserção no mundo do trabalho e da ascensão social e pela identificação com experiências positivas de profissionais da área. Esses elementos podem ser considerados como fundamentais para a identificação social da profissão. ${ }^{14}$

Já os profissionais de enfermagem atuantes no mercado de trabalho percebem não somente a importância dessa área, mas também o quanto a profissão se insere na vida das famílias e o quanto esse fato pode ser positivo ou negativo para os indivíduos cuidados, conforme retratam:

Gostar de cuidar do outro, de ser importante para melhorar a qualidade de vida das pessoas. (ANIS)

Conseguir atender as pessoas e ter o reconhecimento delas não tem preço. (PETÚNIA)

Trabalhar em dois empregos é uma maneira de garantir maior ganho e poder dar melhor condição de vida a minha família. Mas nunca deixando de trabalhar com qualidade. (MARGARIDA)

Enquanto o aluno preocupa-se com o espaço profissional que irá ocupar e as perspectivas profissionais que farão parte do seu futuro quando técnico em enfermagem, o profissional busca encontrar a essência da profissão e a valorização pelo reconhecimento das pessoas assistidas. O falar e o agir de estudantes e profissionais formados diferem em aspectos importantes 
Percepção de discentes, docentes e técnicos em enfermagem a respeito dos eventos adversos |8

relacionados à condição de se perceber como agente de ação e transformação na vida das pessoas. Estabelecer vínculo e se envolver com a situação vivida pelo ser cuidado revelam condições de estabelecimento de processos de cumplicidade e, desta forma, estarem ainda mais próximos desse indivíduo. ${ }^{15}$

Com relação às aspirações futuras sobre a atuação profissional em única ou múltipla jornada de trabalho nas instituições de saúde, 67\% (n=16) dos discentes apontaram que gostariam de atuar em uma única instituição, $29 \%(n=7)$ em duas e $4 \%(n=1)$ como concursado. Os profissionais apontaram que $52 \%(n=13)$ atuam em uma única instituição enquanto que $48 \%$ $(n=12)$ atuam em duas.

A elevada jornada de trabalho na enfermagem é uma realidade, destaca-se que parte desses profissionais trabalha mais de 36 horas semanais, chegando até a 78 horas. Como repercussões dessa carga horária, destacam-se frequentes dores lombares, fadiga/exaustão, chegando a respostas fisiológicas e ao estresse. Nesse sentido, também cuidar dos profissionais que atuam nos serviços de saúde pode ser estratégia fundamental, uma vez que a qualidade do atendimento depende, principalmente, de equipes saudáveis. ${ }^{16}$

$\mathrm{Na}$ entrevista foi possível identificar que a sobrecarga de trabalho pode ser um fator que contribui para a ocorrência de evento adverso durante o processo de cuidar. Atuar nos serviços de saúde, em uma rede de atenção a pessoas fragilizadas e com diferentes tipos de necessidades exige do profissional de enfermagem, para além de conhecimento científico, atitudes seguras e garantia de qualidade no cuidado prestado.

A ocorrência dos eventos adversos está relacionada à sobrecarga de trabalho, que repercute no aumento do número de dias de internação e no risco de óbito dos pacientes. Dessa forma, considerando a influência da sobrecarga de trabalho sobre os processos de adoecimento, destaca-se a importância de melhorar as condições de trabalho dos técnicos em enfermagem. ${ }^{17-18}$ 
Ao desenvolver atividades de enfermagem em unidades de internação e/ou críticas, o cuidar torna-se essencialmente condicionado ao tempo dispensado para cada atividade, bem como aos procedimentos que devem ser realizados. Assim, a sobrecarga de trabalho e a demanda de cuidados de forma simultânea podem contribuir para que o risco de um evento adverso se torne iminente, conforme apresentam as falas a seguir:

Profissão que exige atenção, agilidade no atendimento, responsabilidade nos atos. (DORMIDEIRA)

Tenho muito medo de fazer algo errado, principalmente quando estou cansada e já na segunda jornada de trabalho do dia. (PETÚNIA)

Diante disso, a análise da jornada de trabalho permite identificar o peso que as responsabilidades familiares têm para as mulheres, pois enquanto as jornadas de trabalho dos homens são mais elevadas do que as delas em todos os tipos de famílias, para as mulheres essas variam conforme a presença ou não de dependentes e de parentes que auxiliem nos cuidados com os filhos. Nas famílias em que as mulheres cônjuges têm filhos dependentes e têm parentes no mesmo domicílio, a jornada de trabalho remunerado aumenta em uma hora em relação àquelas que não possuem este apoio. ${ }^{19}$

As falas dos técnicos em enfermagem sobre a jornada de trabalho indicam a necessidade da dupla jornada para complementar a renda familiar, mesmo que seja em diferentes ambientes de atuação.

A carga horária dessa profissão nos proporciona trabalhar em duas instituições. Melhora o salário também. (BROMÉLIA)

Trabalho em quantas instituições puder e atuo de forma particular no cuidado de pessoas em casa e ou internadas. (AZALÉIA ROSADA)

Somado a isso, a pouca atuação do enfermeiro na gestão da equipe de trabalho em alguns setores desestrutura o processo de cuidar seguro. O enfermeiro como gestor de uma unidade de saúde torna-se fundamental no processo do cuidado, uma vez que em sua formação desenvolveu 
Percepção de discentes, docentes e técnicos em enfermagem a respeito dos eventos adversos $\mid 10$

o conhecimento para identificar situações de risco e condicionar as atividades e condutas da equipe de saúde para segurança do cuidado prestado. Entretanto, os participantes do estudo relatam que a supervisão e orientação deste profissional deve ser assumida em sua plenitude, como mediador de saberes e gestor dos processos de trabalho:

Ocorre falta de acompanhamento, diálogo entre os profissionais da enfermagem na equipe, falta de humanização com o paciente e omissão na gestão por parte do enfermeiro. (MARGARIDA)

Queria ter o enfermeiro sempre presente na unidade e participando das decisões importantes. (BROMÉLIA)

A comunicação examinada pelo exposto no contexto da liderança do enfermeiro esboça a dimensão do papel do enfermeiro como líder e fonte central de comunicação nos sistemas de organização da assistência de enfermagem. ${ }^{6}$ A equipe de enfermagem espera do enfermeiro a posição de coordenador do processo de trabalho e profissional presente na unidade e atuante em todas as dimensões do cuidado, contribuindo para a identificação de riscos e sua prevenção. ${ }^{6}$

Nesse sentido, o conhecimento sobre os eventos adversos no cenário dos discentes e dos técnicos em enfermagem repercute de maneira similar. A percepção de que este evento pode repercutir na vida de alguém e de seus familiares preocupa os futuros profissionais, assim como quem está atuando diariamente no cuidado. A falta de atenção e as falhas no processo de formação são citadas como possíveis condicionantes:

Efeitos adversos ou complicação resultante do tratamento médico ou do atendimento realizado por outros profissionais da saúde, muitos vezes, despreparados para o atendimento. (COPO DE LEITE)

Infelizmente isso ainda ocorre. Falta de atenção? Profissionais sobrecarregados? Pressão psicológica nos profissionais? Profissionais despreparados. (ESPORÃO)

São erros nas execuções de procedimentos que causam ou agravam danos irreversíveis à saúde do paciente. (FRÉSIA) 
11 | Machado NCB, Morais EM, Fontana RT, Rodrigues AP, Barros M, Krebs EM

$\mathrm{Na}$ formação, a prática segura é essencial para a segurança dos pacientes e dos profissionais de saúde. Os discentes afirmam ainda que na formação, muitas vezes, falta o amadurecimento necessário para a compreensão dos riscos da profissão e maneiras de assegurar uma postura de segurança:

Falta de atenção, falta de formação profissional e até imaturidade nossa na aproximação com o assunto. (LÍRIO)

A gente não consegue perceber durante a formação o quanto isso é grave, apesar de sempre ser falado. (CRAVO)

A formação prepara o profissional de saúde para não praticar atos inseguros no cuidado das pessoas e o reconhecimento da importância do docente na formação desse profissional repercute na segurança do paciente, uma vez que este tema representa um dos maiores desafios para a excelência da qualidade no serviço de saúde. As condições de trabalho são fatores que comprometem o cuidado e a enfermagem tem participação fundamental nos processos que visam garantir a assistência prestada. ${ }^{20}$

Dentro dessa perspectiva, pode-se afirmar que a ocorrência de eventos adversos no decorrer da assistência pode colocar em risco a vida do indivíduo cuidado. Além disso, o conhecimento das condições de trabalho dos profissionais, bem como as atividades desenvolvidas em duplas jornadas e as condições de saúde merecem atenção, pois podem interferir na assistência. ${ }^{4}$

O processo de cuidar envolve agregar à prática conhecimentos apreendidos durante a formação profissional. Estar em condições de atuar no cuidado de pessoas implica ter clareza das suas responsabilidades, dos seus direitos e seus deveres inerentes a este cuidado.

Assim, os discentes, em sua fala, expressam fragilidade e medo no processo de recuperação do paciente exposto ao evento adverso:

Assumiria o erro, mas com medo, quem sabe “abandonaria a profissão?”. (CAMÉLIA) 
Percepção de discentes, docentes e técnicos em enfermagem a respeito dos eventos adversos | 12

Ficaria nervosa e com medo, mas avisaria a enfermeira averiguassem o meu erro. (TULIPA)

A recuperação do paciente, vítima de algum evento adverso cometido pela equipe de saúde no processo de cuidado, faz parte de um contexto em que a atitude imediata pode resultar positivamente neste desfecho.

Com relação aos profissionais de enfermagem que atuam no cuidado integral dos pacientes, estes demonstram maturidade e envolvimento com o cuidado em todos os aspectos, principalmente, na identificação do seu papel na equipe de trabalho e na atitude frente ao inesperado:

Observar se há alguma intercorrência, aferição dos sinais vitais e comunicar rapidamente a enfermeira. (ORQUIDEA)

Comunicar imediatamente para que o paciente não corra risco de vida, pois o enfermeiro irá comunicar o médico para amenizar o erro. (DORMIDEIRA)

A percepção sobre o evento adverso que cause danos irreversíveis ou morte ao paciente implica em repensar os modos de atenção e estratégias de prevenção deste agravo. As falas sobre o erro e a percepção deste na vida dos discentes e profissionais apresentam tendências similares:

É um erro inaceitável, não devemos cometer erros, quando cometido deve ser investigado. (GARDENIA)

É muito triste, por que é algo que vai marcar para sempre. (TULIPA)

Muito complicado, pois você está colocando a vida de um ser humano em jogo. (AZALÉIA BRANCA)

Acho triste, uma situação que causa danos psicológicos tanto para o paciente como para o profissional. (ACÁCIA)

Penso que deve ser melhor investigado e publicado para que se reflita melhor sobre as condições que o profissional está trabalhando, se está bem física e psicologicamente. (ANIS) 
13 | Machado NCB, Morais EM, Fontana RT, Rodrigues AP, Barros M, Krebs EM

Perceber que a ocorrência de eventos adversos faz parte da organização do trabalho pode contribuir para a discussão e educação permanente da equipe de trabalho no cuidado aos pacientes. Esta postura envolve o reconhecimento daquele que o cometeu, buscando identificar alguma lacuna que contribuiu para esse evento adverso, conforme as falas dos discentes:

\section{A responsabilidade do erro é do profissional que comete. (GARDENIA)}

Há ninguém, pois todos nós somos humanos e ninguém está livre de errar. Mas precisamos ter o máximo de seriedade com que fazemos, para evitar erros, já que dependendo o erro pode até custar uma vida. (COPO DE LEITE)

Os profissionais percebem o evento adverso como um processo transversal, que atinge toda a equipe, inclusive o enfermeiro que supervisiona e orienta sua equipe de trabalho:

Todos têm uma parcela de culpa em um erro, como equipe todos tem que ter responsabilidade. (MAGNÓLIA)

Nesse contexto, os docentes têm papel fundamental na formação profissional e a capacidade de fazer com que o discente se aproprie de conhecimentos, possibilitando discussões e reflexões essenciais para o processo de trabalho dos futuros profissionais. ${ }^{21}$ Com isso, é necessário o olhar docente direcionando o aluno de forma consciente da sua importância no processo de cuidar, ${ }^{22}$ com a sua presença constante nas atividades práticas, despertando no discente um senso apurado para situações de risco que podem ocorrer:

As falhas na formação profissional podem contribuir para os erros, por isso o aluno sempre deve ser alertado. (VIOLETA)

É preciso estar muito próximo do aluno. (CRISANTEMO)

Existem fatores individuais que podem agravar situações de risco para o erro de enfermagem. (ROSA)

Pensar na formação de sujeitos, ainda imaturos, para as situações que envolvem a vida e a saúde das pessoas pode ser um agravante, que merece atenção especial por parte dos docentes, 
Percepção de discentes, docentes e técnicos em enfermagem a respeito dos eventos adversos | 14

uma vez que o conhecimento não atinge a todos da mesma maneira. Neste sentido, nortear a formação para um processo de atenção para a prevenção e identificação dos eventos adversos no cuidado torna-se essencial.

\section{Considerações finais}

Os eventos adversos representam uma preocupação no cenário de saúde e envolve os mais diversos profissionais. A discussão sobre os eventos adversos na enfermagem condiciona a uma postura de atuação preventiva com aproximação das instituições de ensino aos serviços de saúde.

Conforme os resultados são possíveis destacar que a sobrecarga de trabalho e a demanda de cuidados podem contribuir para a ocorrência de algum evento adverso. Além disso, a falta de atenção e as falhas no processo de formação são citadas como possíveis condicionantes à essa problemática.

A identificação de possíveis falhas no processo de formação e de educação permanente em saúde contribui para a prática docente e para a gestão do enfermeiro diante da sua equipe de trabalho. O presente estudo implica em ampliar e aprofundar as pesquisas que abordem a atuação do técnico em enfermagem, uma vez que a maioria dos estudos destaca apenas a prática do enfermeiro.

Para o ensino, percebe-se que as diferenças cruciais entre discentes e profissionais formados no que se refere a eventos adversos estabelece uma porta de comunicação essencial para (re) descobrir formas de ensinar enfermagem de maneira segura e de qualidade. Assim, contribui-se para a melhoria do ensino dessa classe profissional, repercutindo na sua atuação, com vistas a minimizar ou anular a ocorrência de eventos adversos.

A limitação do estudo envolveu a dificuldade de abordagem dos técnicos em enfermagem no cotidiano de trabalho, devido ao elevado número de atribuições nas unidades hospitalares. Ressalta-se a necessidade de realização de novos estudos que ampliem os campos de coleta de dados para outros níveis de atenção à saúde e instituiçõos de ensino, incluindo demais profissionais que compõem a equipe de saúde. 


\section{Referências}

1. Moro L. Atuação docente no processo de ensino-aprendizagem: os desafios pedagógicos nas diversidades socioculturais. Só Pedagogia [Internet]. 2019 [acesso em 2019 out 10]. Disponível em: https://www.pedagogia.com.br/artigos/atuacaodocente/index.php?pagina=0

2. Souza CS, Dourado L. Aprendizagem baseada em problemas (ABP): um método de aprendizagem inovador para o ensino educativo. Holos [Internet]. 2015 [acesso em 2018 jan 30];31(5):182-200. Disponível em: https://doi.org/10.15628/holos.2015.2880

3. Cónsul-Giribet M, Medina-Moya JL. Pontos fortes e deficiências da aprendizagem baseada em problemas sob a perspectiva profissional de enfermeiras. Rev Latinoam Enferm [Internet]. 2014 set-out [acesso em 2018 jan 30];22(5):724-30. Disponível em: http://www.scielo.br/pdf/rlae/v22n5/pt_0104-1169rlae-22-05-00724.pdf

4. World Health Organization (WHO), World Aliance for Patient Safety. Taxonomy: the conceptual framework for the international classification for patient safety. Genève: WHO; 2009.

5. Duarte SCM, Stipp MAC, Silva MM, Oliveira FT. Eventos adversos e segurança na assistência de enfermagem. Rev Bras Enferm [Internet]. 2015 jan-fev [acesso em 2019 out 10];68(1):144-54. Disponível em: http://www.scielo.br/pdf/reben/v68n1/0034-7167-reben-68-01-0144.pdf

6. Treviso P, Peres SC, Silva AD, Santos AA. Competências do enfermeiro na gestão do cuidado. Rev Adm Saúde [Internet]. 2017 out-dez [acesso em 2018 fev 02];17(69). Disponível em: http://www.cqh.org.br/ojs-2.4.8/index.php/ras/article/view/59/77

7. Agência Nacional de Vigilância Sanitária (ANVISA). Assistência segura: uma reflexão teórica aplicada à prática. Brasília (DF): ANVISA; 2017.

8. Fontana RT, Wolf J, Rodrigues FCP, Castro LM. Análise documental da mídia escrita sobre eventos adversos ocorridos na prática da enfermagem. Rev Enferm UFPE On Line [Internet]. 2015 maio [acesso em 2018 fev 02];9(Supl 4):8103-10. Disponível https://periodicos.ufpe.br/revistas/revistaenfermagem/article/view/10565/11499

9. Forte ECN, Machado FL, Pires DEP. A relação da enfermagem com os erros de medicação: uma revisão integrativa. Cogitare Enferm [Internet]. 2016 [acesso em 2018 fev 04];21(N Esp):01-10. Disponível em: http://www.saude.ufpr.br/portal/revistacogitare/wp-content/uploads/sites/28/2016/09/43324-184651-1PB.pdf

10. Agência Nacional de Vigilância Sanitária (ANVISA). Notivisa: notificações em vigilância sanitária [Internet]; 2019 [acesso em 2019 fev 04]. Disponível em: http://portal.anvisa.gov.br/notivisa

11. Minayo MC. Pesquisa social: teoria, método e criatividade. Rio de Janeiro (RJ): Vozes; 2016. 
Percepção de discentes, docentes e técnicos em enfermagem a respeito dos eventos adversos | 16

12. Brasil. Ministério da Saúde. Conselho Nacional de Saúde. Resolução no 466, de 12 de dezembro de 2012 [Internet]. 2012 [acesso em 2018 fev 04]. Disponível em: https://bvsms.saude.gov.br/bvs/saudelegis/cns/2013/res0466_12_12_2012.html

13. Splendor VL, Roman AR. A mulher, a enfermagem e o cuidar na perspectiva de gênero. Rev Contexto Saúde [Internet]. 2013 maio [acesso em 2018 fev 10];3(4):31-44. Disponível em: https://www.revistas.unijui.edu.br/index.php/contextoesaude/article/view/1268

14. Teodosio SSC, Padilha MI. "Ser enfermeiro": escolha profissional e a construção dos processos identitários (anos 1970). Rev Bras Enferm [Internet]. 2016 [acesso em 2018 fev 10];69(3):401-7. Disponível em: http://www.scielo.br/pdf/reben/v69n3/0034-7167-reben-69-03-0428.pdf

15. Paiva ACPC, Salimena AMO. O olhar da mulher sobre os cuidados de enfermagem ao vivenciar o câncer de mama. HU Rev [Internet]. 2016 jan-jun [acesso em 2018 mar 20];42(1):11-7. Disponível em: https://hurevista.ufjf.emnuvens.com.br/hurevista/article/viewFile/2275/843

16. Dalri RCMB, Silva LA, Mendes AMOC, Robazzi LMCC. Carga horária de trabalho dos enfermeiros e sua relação com as reações fisiológicas do estresse. Rev Latinoam Enfermagem [Internet]. 2014 nov-dez [acesso em 2018 mar 20];22(6):959-65. Disponível em: http://www.scielo.br/pdf/rlae/v22n6/pt_0104-1169rlae-22-06-00959.pdf

17. Novaretti MCZ, Santos EV, Quiterio LM, Daud-Gallotti RM. Sobrecarga de trabalho da enfermagem e incidentes e eventos adversos em pacientes internados em UTI. Rev Bras Enferm [Internet]. 2014 [acesso em 2018 abr 15];67(5):692-9. Disponível em: http://www.scielo.br/pdf/reben/v67n5/0034-7167reben-67-05-0692.pdf

18. Vieira GLC. Satisfação e sobrecarga de trabalho entre técnicos de enfermagem de hospitais psiquiátricos. Rev Port Enferm Saúde Mental [Internet]. 2017 jun [acesso em 2018 abr 15];17. Disponível em: http://www.scielo.mec.pt/pdf/rpesm/n17/n17a06.pdf

19. Moser L, Dal Prá KR. Os desafios de conciliar trabalho, família e cuidados: evidências do "familismo" nas políticas sociais brasileiras. Textos Contextos (Porto Alegre) [Internet]. 2016 ago-dez [acesso em 2018 abr 16];15(2):382-92. Disponível em: http://revistaseletronicas.pucrs.br/ojs/index.php/fass/article/view/21923/15299

20. Cavalcante AKCB, Rocha RC, Nogueira LT, Avelino FVSD, Rocha SS. Cuidado seguro ao paciente: contribuições da enfermagem. Rev Cuba Enferm [Internet]. 2015 [acesso em 2018 abr 16];31(4). Disponível em: http://www.revenfermeria.sld.cu/index.php/enf/article/view/907

21. Canever BP, Prado ML, Gome DC, Jesus BH, Backes VMS. Consciência de mundo ingênua na prática pedagógica de docentes da área da saúde. Texto Contexto Enferm [Internet]. 2017 [acesso em 2018 abr 20];26(2):1-9. Disponível em: http://www.scielo.br/pdf/tce/v26n2/pt_0104-0707-tce-26-02-e3340015.pdf 
17 | Machado NCB, Morais EM, Fontana RT, Rodrigues AP, Barros M, Krebs EM

22. Ramos TK, Nietsche EA, Cogo SB, Cassenote LG, Böck A, Martins FS. Estagio curricular supervisionado e a formação do enfermeiro: atividades desenvolvidas. Rev Enferm UFSM [Internet]. 2018 jan-mar [acesso em 2018 maio 02];8(1):59-71. Disponível em: https://periodicos.ufsm.br/reufsm/article/view/28124/pdf

\section{Autor correspondente}

Neiva Claudete Brondani Machado

E-mail: neiva.machado@iffarroupilha.edu.br

Endreço: Instituto Federal de Educação, Ciência e Tecnologia Farroupilha - Campus Santo Ângelo. RS 218 -

Km 5 - Indúbras - Santo Ângelo/RS Telefone: (55) 3931-3900

CEP: $98806-700$

\section{Contribuições de Autoria}

\section{1 - Neiva Claudete Brondani Machado}

Concepção e planejamento do projeto de pesquisa, análise e interpretação dos dados, redação e revisão crítica do artigo.

\section{2 - Edinara Moraes Morais}

Concepção do projeto de pesquisa, análise e interpretação dos dados e revisão crítica do artigo.

\section{3 - Rosane Teresinha Fontana}

Análise e interpretação dos dados, redação e revisão crítica do artigo.

\section{4 - Andressa Peripolli Rodrigues}

Análise e interpretação dos dados, redação e revisão crítica do artigo.

\section{5 - Miriam de Barros}

Concepção e planejamento do projeto de pesquisa, análise e interpretação dos dados e revisão crítica do artigo.

\section{6 - Elizabet Marta Krebs}

Análise e interpretação dos dados, redação e revisão crítica do artigo.

\section{Como citar este artigo}

Machado NCB, Morais EM, Fontana RT, Rodrigues AP, Barros M, Krebs EM. Percepção de discentes, docentes e técnicos em enfermagem a respeito dos eventos adversos. Rev. Enferm. UFSM. 2020 [Acesso em: Anos Mês Dia]; vol.10 e: 1-18. DOI:https://doi.org/10.5902/2179769233486 